\title{
Variações da pressão intracraniana durante manobra de expansão pulmo- nar em pacientes com trauma cranio- encefálico grave, monitorizados em unidade de terapia intensiva
}

\author{
Intracranial pressure changes during pulmonary expansion \\ maneuver in patients with severe traumatic brain injury, \\ monitored at the unity of intensive therapy
}

Silvia A. Ferreira', Vera L. Israel², Luiz R. Aguiar³

\begin{abstract}
RESUMO
A fisioterapia dispõe de recursos específicos para a reabilitação em unidade de terapia intensiva (UTI), assim como nos pacientes com traumatismo cranioencefálico (TCE) grave. Entre essas técnicas, destaca-se a manobra de expansão pulmonar, que é uma técnica cinesioterapêutica, a qual tem função de mobilizar secreção pulmonar, prevenir e tratar atelectasias. Modelo do estudo: $O$ estudo foi classificado como pesquisa de campo (experimental), baseada em avaliação da PIC durante e após a manobra de expansão pulmonar. Objetivo: Verificar a influência da manobra fisioterapêutica de expansão pulmonar nos valores da pressão intracraniana (PIC) em pacientes com TCE grave. Metodologia: A pesquisa foi aprovada pelo Comitê de Ética e Pesquisa da PUCPR, com o parecer 1455. Foi realizada na unidade de terapia intensiva (UTI) do Hospital Universitário Cajuru, Curitiba-PR. Participaram do estudo 15 pacientes de ambos os sexos, com TCE grave e com faixa etária entre 18 e 50 anos. As variáveis monitorizadas foram a PIC, a pressão arterial média (PAM) e a pressão de perfusão cerebral (PPC). Resultados: Durante a aplicação do protocolo de pesquisa observou-se que a PAM e a PPC mantiveram-se dentro ou próximos da normalidade com mínimas variações, enquanto que a PIC evolui com variação aproximada de $1 \mathrm{mmHg}$ retornando aos valores iniciais. As médias da PIC no 1을, $2^{\circ}$ dia e $3^{\circ}$ dia foram $5,42( \pm 4,69)$ $\mathrm{mmHg}, 6,71( \pm 6,84) \mathrm{mmHg}$ e $5,60( \pm 4,33) \mathrm{mmHg}$, com significância estatística respectivamente $p<0,001$, $p=0,008$ e $p=0,001$. Conclusões: A manobra de expansão pulmonar pode ser realizada em pacientes com TCE grave, estáveis hemodinamicamente e com PIC abaixo de $20 \mathrm{mmHg}$.
\end{abstract}

Palavras-chave: Fisioterapia (Especialidade). Serviço Hospitalar de Fisioterapia. Terapia Respiratória. Pressão Intracraniana. Trauma Craniocerebral.

1. Fisioterapeuta, Mestre em Tecnologia em Saúde pela PUCPR; Fisioterapeuta do Serviço de Fisioterapia do Hospital Universitário Cajuru; Curitiba - PR.

2. Fisioterapeuta, Doutora em Educação Especial / UFSCAR, Professora da UFPR-Litoral; Matinhos - PR.

3. Médico, Doutor em Medicina / UNIFESP, Professor PPGTSPUCPR, Médico Neurocirurgião do Hospital Universitário Cajuru; Curitiba - PR
Correspondência:

Silvia Aparecida Ferreira Hospital Universitário Cajuru - Serviço de Fisioterapia Avenida São José, 300 - Cristo Rei 80050-350 - Curitiba / PR E-mail: silvia.ferreira@pucpr.br

Artigo recebido em 03/09/2008 Aprovado em 10/03/2010 


\section{Introdução}

Anatomicamente, o cérebro constitui-se por volumes intracranianos, os quais são compreendidos pelo parênquima cerebral (80\%), pelo sangue (10\%) e pelo líquido cefalorraquidiano (10\%). Estes volumes permanecem constantemente equilibrados em pessoas sem trauma. ${ }^{1}$ Nos casos dos pacientes com traumatismo cranioencefálico (TCE) grave é comum o desequilíbrio entre qualquer um desses volumes, ocasionando alteração dos níveis da pressão intracraniana (PIC). A monitorização eletrônica da PIC tornou-se possível no início de 1960, quando Lundberg mediu a pressão do fluído ventricular cerebral por meio de um cateter de polietileno acoplado a um transdutor de pressão. ${ }^{2}$

A escala de coma de Glasgow (GCS) infere que se pode classificar o grau do TCE como: leve, moderado e grave. ${ }^{3}$ Paciente com TCE grave e GCS com escore igual ou inferior a 08 indica-se à monitorização de PIC. ${ }^{4}$ Com o uso desta monitorização contínua, em conjunto com a GCS, é possível traçar o possível prognóstico do paciente. ${ }^{5-7}$

$\mathrm{O}$ aumento da PIC pode ser decorrente dos seguintes mecanismos: lesões expansivas na caixa craniana; obstrução do fluxo de saída do líquido cefalorraquidiano; aumento de líquido no espaço intersticial e/ou intracelular; engurgitamento. ${ }^{8-9}$ Uma complicação importante em curto prazo é o edema cerebral do tecido lesado, o qual atinge seu grau máximo em 72 horas após o trauma. ${ }^{5}$ Desta forma, é primordial a monitorização precoce da PIC nos pacientes com TCE grave.

Os motivos para monitor a PIC são minimizar os possíveis efeitos deletérios do TCE, diminuir o índice de mortalidade e morbidade, devido ao risco de hipertensão intracraniana (HIC), que é letal, avaliar efeitos do tratamento com objetivo de diminuir a PIC e limitar sua variação. ${ }^{1,10-11}$

$\mathrm{O}$ valor considerado normal para a PIC em pacientes com TCE na UTI pode ser aceito em até 20 mmHg. A PIC média normal é considerada entre 10$15 \mathrm{mmHg}$, pressão que não causa comprometimento cerebral, isto é, não tem risco de óbito para o paciente. A elevação da PIC de $15-20 \mathrm{mmHg}$ comprime os leitos capilares e compromete a microcirculação, porém, neste caso, apesar das alterações fisiológicas, não causa lesão cerebral, ou seja, considera-se uma pressão limite. Abaixo de $20 \mathrm{mmHg}$, ocorre adequada perfusão cerebral na vigência de PAM normal e impede a herniação cerebral. ${ }^{10,12}$ Quando o valor da PIC encontra-se acima de $20 \mathrm{mmHg}$, este é denominado de HIC. Nesse caso, o paciente necessita de um cuidado específico para normalizar a PIC. Na vigência do quadro de HIC, deve-se atentar também aos valores da PAM e PPC.

A PAM ideal é considerada entre $60-180 \mathrm{mmHg}$. No entanto, quando a PAM é inferior a $60 \mathrm{mmHg}$, o cérebro fica isquêmico, devido ao aumento da $\mathrm{PaCO}_{2}$ e queda da $\mathrm{PaO}_{2}$. Na vigência deste quadro ocorre uma vasodilatação cerebral, a qual promove engurgitamento venoso e cerebral e, consequentemente, elevação da PIC. Se a elevação da PAM for superior a $180 \mathrm{mmHg}$, ocorrerá o aumento do FSC, evoluindo para uma vasoconstrição, queda da pressão intravascular e drenagem venosa. ${ }^{1,9,13-14}$ Estas geram a elevação do volume sanguíneo cerebral e da PIC.

A PPC15 é igual a diferença entre a PAM e PIC, representada na Equação 1:

$$
\mathrm{PPC}=\mathrm{PAM}-\mathrm{PIC}
$$

A PPC permanece em constante acomodação, sendo esta decorrente da variação dos volumes cerebrais, mantendo-se acima de $60 \mathrm{mmHg}$. ${ }^{1,13-16}$. Quando a PPC está abaixo de $60 \mathrm{mmHg}$ e a PIC acima de $20 \mathrm{mmHg}$, existe uma tendência no decréscimo da complacência, com insuficiência do mecanismo de autorregulação. ${ }^{17}$

O estudo foi classificado como pesquisa de campo baseado na coleta de dados em pacientes com TCE grave, com o objetivo de avaliar a influência da manobra fisioterapêutica de expansão pulmonar nos valores da PIC, PPC e pressão arterial média (PAM) em paciente com TCE grave, em unidade de terapia intensiva (UTI).

\section{Material e Método}

Esta pesquisa foi fundamentada em uma visão teórica-prática, classificada como uma pesquisa de campo. O estudo foi classificado como quantitativo e qualitativo, sendo aprovado pelo Comitê de Ética em Pesquisa - PUCPR, com o parecer $n^{\circ} 1455$. De acordo com as normas da mesma, o estudo foi iniciado quando autorizada a inclusão do paciente na pesquisa pelo familiar responsável, mediante a assinatura do termo de consentimento livre e esclarecido.

Participaram do estudo quinze pacientes de ambos os sexos com TCE grave. Os fatores de inclusão foram: pacientes com TCE grave, faixa etária entre 18 e 50 anos, internados na UTI, com intubação 
endotraqueal, dependentes de assistência ventilatória mecânica, com monitorização de PIC, sedados e/ou curarizados, ausência de fratura de arcos costais, ausência de trauma abdominal, cardiopatias e/ou ausência de doença pulmonar obstrutiva crônica associada. Já os fatores de exclusão foram pacientes com morte encefálica, em respiração espontânea ou no modo de ventilação por pressão de suporte e valor da PIC igual ou superior a $20 \mathrm{mmHg}$.

As variáveis coletadas foram PIC, PAM e PPC. Para o desenvolvimento da pesquisa foi necessário o monitor de PAM, da marca HP® (Hewlett Packard), modelo Viridia 24 e o monitor de PIC com sensor de fibra óptica da marca Spiegelberg e modelo Aesculap.

Os pacientes foram acompanhados durante três dias consecutivos, a partir do primeiro dia de implantação do cateter de PIC, sendo a manobra aplicada pelo mesmo fisioterapeuta durante todo protocolo.

Para iniciar o protocolo da avaliação da PIC durante a manobra de expansão pulmonar de compressão-descompressão torácica, o paciente foi posicionado no leito de acordo com as seguintes orientações:

- Paciente em decúbito dorsal (DD) com cabeceira elevada a 30 graus, para facilitar retorno venoso cerebral, diminuindo assim a estase sanguínea. Este posicionamento ajuda a prevenir a HIC; , $^{4,8,16,18,19}$

- Cabeça em linha reta evitando rotação e flexão cervical, com a finalidade de evitar compressão e distorção das veias jugulares; ${ }^{20}$

- Membros inferiores com flexão de quadril no máximo de $90^{\circ}$, devido ao risco de limitar a drenagem venosa de membros inferiores; ${ }^{21}$

- Vias aéreas pérveas, livres e ventiladas. ${ }^{22}$

Neste estudo foi eleita uma manobra de expansão/re-expansão pulmonar, que conforme Jerre et al. ${ }^{23}$ são o uso de procedimentos que aumentem a pressão e/ou volume alveolar, o que acaba promovendo a expansão de unidades alveolares colabadas.

Em UTI, as indicações clínicas para a utilização das manobras de expansão pulmonar são nos casos de pneumotórax, derrame pleural, atelectasias, shunt pulmonar, pós-operatório de cirurgias toracoabdominais, hipoxemia, senilidade, restrição prolongada de pacientes ao leito e toda alteração que evolua para restrição ventilatória. ${ }^{23-26}$ Estas técnicas são frequentemente utilizadas em ambientes hospitalares.

O objetivo da manobra eleita na pesquisa é recrutar a ventilação alveolar, promover a eliminação de dióxido de $\mathrm{CO}_{2}$, diminuir a resistência das vias aéreas e o trabalho respiratório, expandirem as áreas pulmonares com atelectasias, mobilizar secreção pulmonar, e melhorar a oxigenação arterial, o volume corrente, a capacidade pulmonar, o volume residual inspiratório e a complacência dinâmica. ${ }^{24-27}$ Destacase que, em geral, as manobras de expansão pulmonar visam principalmente melhorar o aporte de $\mathrm{O}_{2}$, mediante expansão das áreas ou regiões de maior resistência nos pulmões. ${ }^{28,29}$

Esta manobra foi descrita por McCarren, Alison e Herbert ${ }^{30,31}$ como vibração, que é definida como a aplicação manual de movimentos combinados de oscilação com compressão no tórax do paciente durante a expiração. Técnica utilizada por fisioterapeutas principalmente para remoção de secreção pulmonar e indicada para paciente com doenças respiratórias. ${ }^{30-32}$ As variações fisiológicas constatadas num estudo realizado por McCarren, Alison e Herbert, ${ }^{30}$ onde foram avaliados sete fisioterapeutas que aplicaram a técnica de vibração em três pacientes, demonstrou que o pico de pressão intrapleural durante a manobra de vibração no momento da compressão aumentou $16 \%$, enquanto que durante a oscilação foi de $18 \%$. Tanto na compressão como na oscilação, ambos componentes da vibração, aumentam a pressão intrapleural.

A manobra de compressão-descompressão torácica é uma técnica manual, onde é realizada uma força na parede do tórax do paciente com as mãos do fisioterapeuta. Na expiração é realizada a compressão torácica e na inspiração é feita uma descompressão súbita. ${ }^{32,33,34}$ Isto gera uma elevação no fluxo da expiração e uma variação súbita do fluxo durante a inspiração, o que favorece tanto a reexpansão pulmonar quanto a desobstrução das vias aéreas. ${ }^{35}$ Esta técnica foi aplicada durante 15 repetições por dia de protocolo. A realização da manobra de expansão pulmonar de compressão-descompressão torácica segue a sequência das Figuras 1, 2, 3 e 4.

Os dados foram coletados em seis fases:

Fase I: anotação inicial dos dados no período anterior da aplicação da manobra;

Fase II: anotação dos dados durante a aplicação da manobra de expansão pulmonar;

Fase III: anotação dos dados 1 minuto pós-manobra; Fase IV: anotação dos dados 5 minutos pós-manobra;

Fase V: anotação dos dados 15 minutos pós-manobra;

Fase VI: finalizando com anotação de dados 30 minutos pós-manobra. 


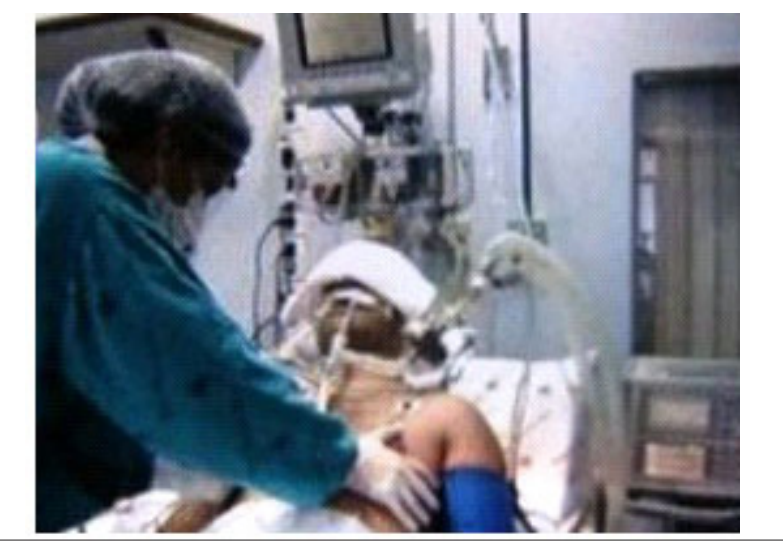

Figura 1: Primeira etapa: o fisioterapeuta se posiciona ao lado do leito do paciente

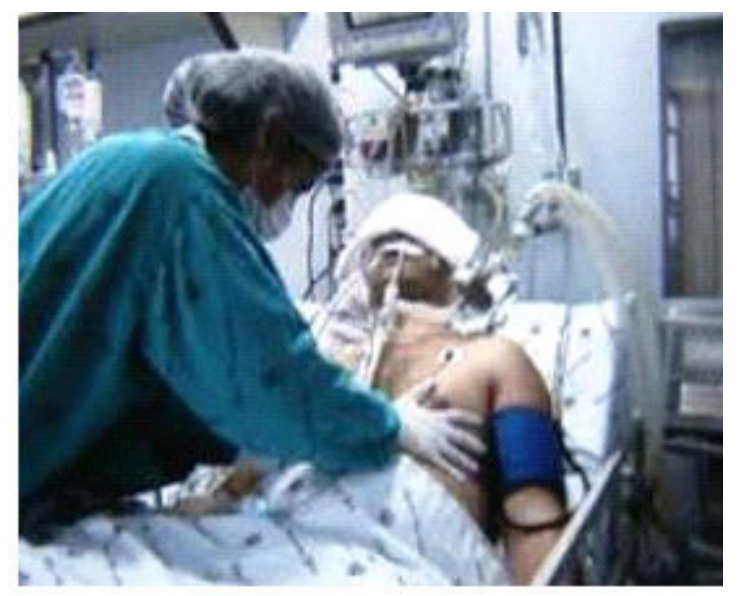

Figura 3: Terceira etapa: o fisioterapeuta realiza a compressão torácica durante a fase expiratória

A análise estatística foi feita através da variância (ANOVA). O nível de significância adotado foi $5 \%$.

\section{Resultados}

A pesquisa foi constituída de 15 pacientes com média de idade de $29( \pm 13)$ anos, destes $13 \%$ do sexo feminino e $87 \%$ do sexo masculino. As diferenças existentes entre os 15 pacientes foram também observadas com cuidado, apesar dos quadros clínicos apresentarem etiologias diversas quanto à origem do trauma e aos tipos de lesão (Tabela 1). A incidência maior do tipo de trauma foi queda de nível $(46,7 \%)$, seguida por colisão de automotivos $(33,3 \%)$ e, por agressão, atropelamento e corte contuso $(20,1 \%)$. Inicialmente foram calculadas as médias do PIC, PAM e PPC nos distintos dias de aplicação do protocolo (Tabela 2).

$\mathrm{O}$ valor médio da $\mathrm{PIC}$ no $1^{\circ}$ dia do protocolo foi de 5,42 $( \pm 4,69) \mathrm{mmHg}$. Se comparando a PIC no

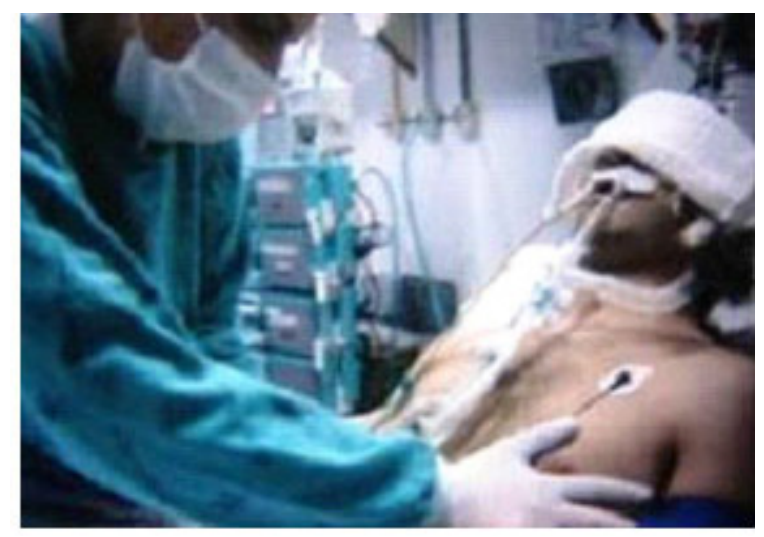

Figura 2: Segunda etapa: o fisioterapeuta posiciona as mãos sobre os últimos arcos costais do paciente

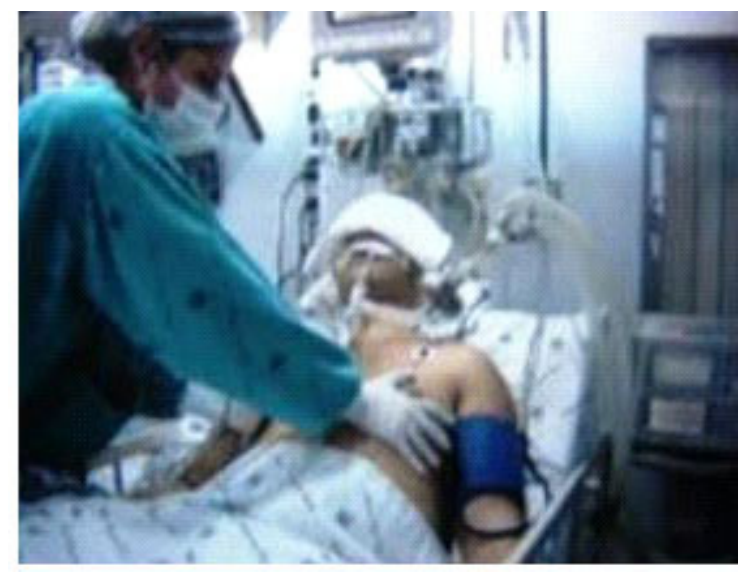

Figura 4 : Quarta etapa: o fisioterapeuta realiza a descompressão torácica no início da fase inspiratória e retorna

\section{Tabela 1}

Descrição do perfil dos pacientes (Mecanismo e tipo do trauma)

\begin{tabular}{cll}
\hline Paciente & Trauma & TC* \\
\hline 1 & Queda de nível & Swelling difusa \\
2 & Queda de nível & HED \\
3 & Colisão & HED e Swelling difusa \\
4 & Queda de nível & HSA \\
5 & Colisão & HED \\
6 & Atropelamento & HED \\
7 & Colisão & HSA \\
8 & Agressão & HSD, HSA e Swelling difusa \\
9 & Queda de nível & HSD \\
10 & Queda de nível & HED \\
11 & Queda de nível & HSA \\
12 & Colisão & HSD \\
13 & Colisão & HSA \\
14 & Corte contuso & HSD \\
15 & Queda de nível & HED \\
\hline
\end{tabular}

*TC: Tomografia Computadorizada; HED: Hematoma Extradural; HSA: Hemorragia Subaracnóidea; HSD: Hematoma Subdural. 


\section{Tabela 2}

Pressão intracraniana (PIC), pressão arterial média (PAM) e pressão de perfusão cerebral (PPC), pré, durante e após $(1,5,15$ e 30 minutos) da manobra de expansão pulmonar.

\begin{tabular}{|c|c|c|c|}
\hline & $1^{\circ}$ Dia & $2^{\circ}$ Dia & $3^{\circ}$ Dia \\
\hline Momento & Média \pm DP & Média \pm DP & Média $\pm \mathrm{DP}$ \\
\hline \multicolumn{4}{|l|}{ PIC } \\
\hline Pré & $6,62 \pm 4,54$ & $7,91 \pm 7,07$ & $6,56 \pm 4,77$ \\
\hline Imediato & $3,64 \pm 5,31$ & $5,58 \pm 7,41$ & $3,61 \pm 3,38$ \\
\hline $1 \mathrm{~min}$ & $3,59 \pm 4,50$ & $5,51 \pm 7,01$ & $4,23 \pm 4,02$ \\
\hline $5 \mathrm{~min}$ & $5,06 \pm 4,06$ & $7,54 \pm 7,25$ & $6,28 \pm 5,31$ \\
\hline $15 \mathrm{~min}$ & $6,42 \pm 4,89$ & $6,71 \pm 6,05$ & $6,37 \pm 4,47$ \\
\hline $30 \mathrm{~min}$ & $7,16 \pm 4,69$ & $7,03 \pm 6,26$ & $6,56 \pm 4,02$ \\
\hline \multicolumn{4}{|l|}{ PAM } \\
\hline Pré & $73,7 \pm 12,04$ & $84,6 \pm 10,80$ & $89,5 \pm 8,97$ \\
\hline Imediato & $74,3 \pm 12,31$ & $86,6 \pm 14,53$ & $87,9 \pm 9,89$ \\
\hline $1 \mathrm{~min}$ & $73,5 \pm 12,92$ & $82,8 \pm 13,06$ & $87,7 \pm 11,38$ \\
\hline $5 \mathrm{~min}$ & $72,6 \pm 13,86$ & $82,1 \pm 11,40$ & $85,3 \pm 10,49$ \\
\hline $15 \mathrm{~min}$ & $74,9 \pm 11,49$ & $81,1 \pm 11,32$ & $87,0 \pm 9,97$ \\
\hline $30 \mathrm{~min}$ & $72,9 \pm 14,89$ & $83,0 \pm 10,87$ & $86,0 \pm 9,17$ \\
\hline \multicolumn{4}{|l|}{ PPC } \\
\hline Pré & $67,07 \pm 13,24$ & $75,59 \pm 14,26$ & $76,32 \pm 24,8$ \\
\hline Imediato & $70,30 \pm 13,57$ & $81,41 \pm 15,47$ & $73,21 \pm 25,98$ \\
\hline $1 \mathrm{~min}$ & $69,59 \pm 14,30$ & $77,05 \pm 15,19$ & $76,36 \pm 27,85$ \\
\hline $5 \min$ & $67,34 \pm 14,98$ & $74,54 \pm 13,09$ & $73,88 \pm 23,62$ \\
\hline $15 \mathrm{~min}$ & $63,15 \pm 23,87$ & $74,39 \pm 12,96$ & $74,78 \pm 24,54$ \\
\hline $30 \mathrm{~min}$ & $65,64 \pm 16,30$ & $76,37 \pm 10,82$ & $73,46 \pm 24,52$ \\
\hline
\end{tabular}

*unidades: mmHg; DP: desvio padrão

momento pré-manobra com a PIC após 30 minutos do término da manobra de expansão pulmonar (Gráfico 1), observamos acréscimo aproximado de $1 \mathrm{mmHg}$ na pressão (de 6,62 \pm 4,54 para 7,16 $\pm 4,69 \mathrm{mmHg}$ ), tendo significância estatística neste dia de $\mathrm{p}<0,001$.

Entretanto, no $2^{\circ}$ dia a média da PIC foi igual $6,71( \pm 6,84) \mathrm{mmHg}$. Observou-se discreto decréscimo da PIC se comparando a pressão pré-manobra com a pressão após 30 minutos do término da manobra (de 7,91 \pm 7,07 para 7,03 $\pm 6,26 \mathrm{mmHg}$ ) (Gráfico 2). Também a PIC apresentou significância estatística de $\mathrm{p}=0,008$.
No $3^{\circ}$ dia, a média da PIC foi 5,60 $( \pm 4,33)$ $\mathrm{mmHg}$. Os valores da PIC, nesta data, foram os menores durante todo o protocolo, se comparado à média de pressão pré e pós (de 6,56 4,77 para 6,56 \pm $4,02 \mathrm{mmHg}$ ) (Gráfico 3). A significância estatística obtida da PIC foi $\mathrm{p}=0,001$.

Os demais dados como PAM e PPC mantiveram valores próximos da normalidade durante os três dias de protocolo. A PAM com a média de 81,42 $( \pm 11,63) \mathrm{mmHg}$ e a PPC com média de $72,80( \pm 18,30)$ $\mathrm{mmHg}$. 


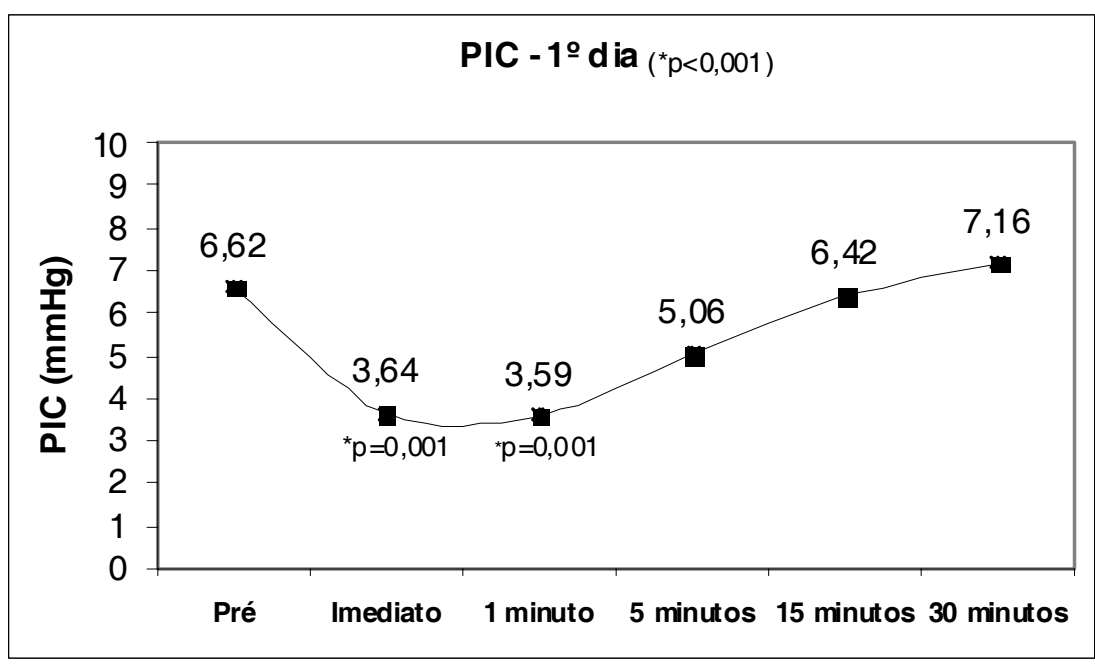

Gráfico 1: Médias da PIC dos 15 pacientes nos distintos momentos da avaliação referente ao 1 dia.

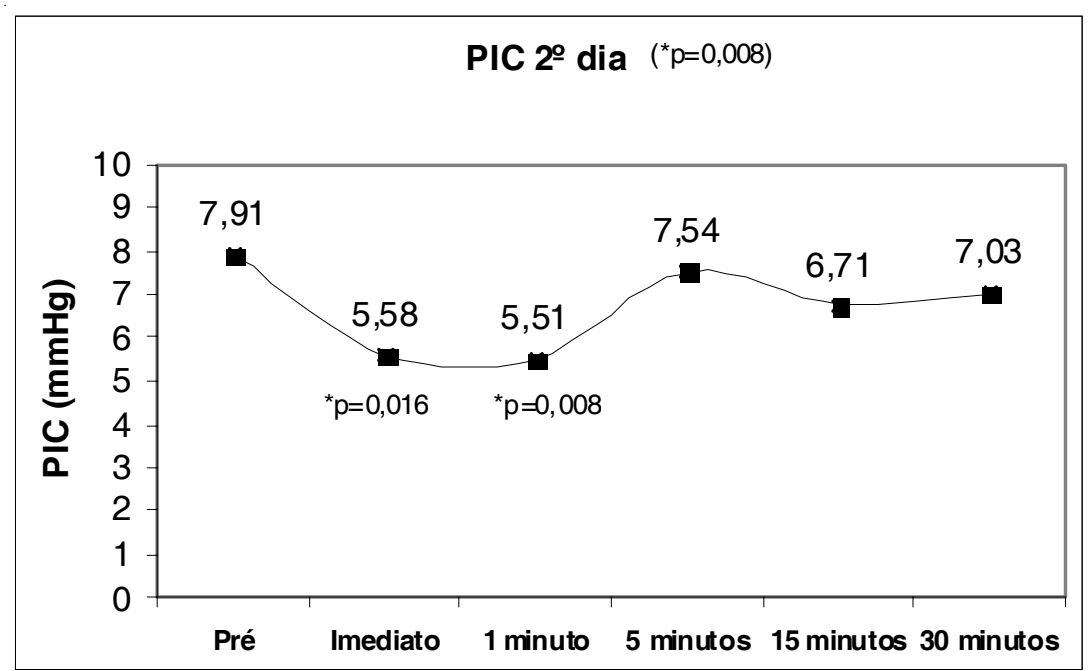

Gráfico 2: Médias da PIC dos 15 pacientes nos distintos momentos da avaliação referente ao $2^{\circ}$ dia.

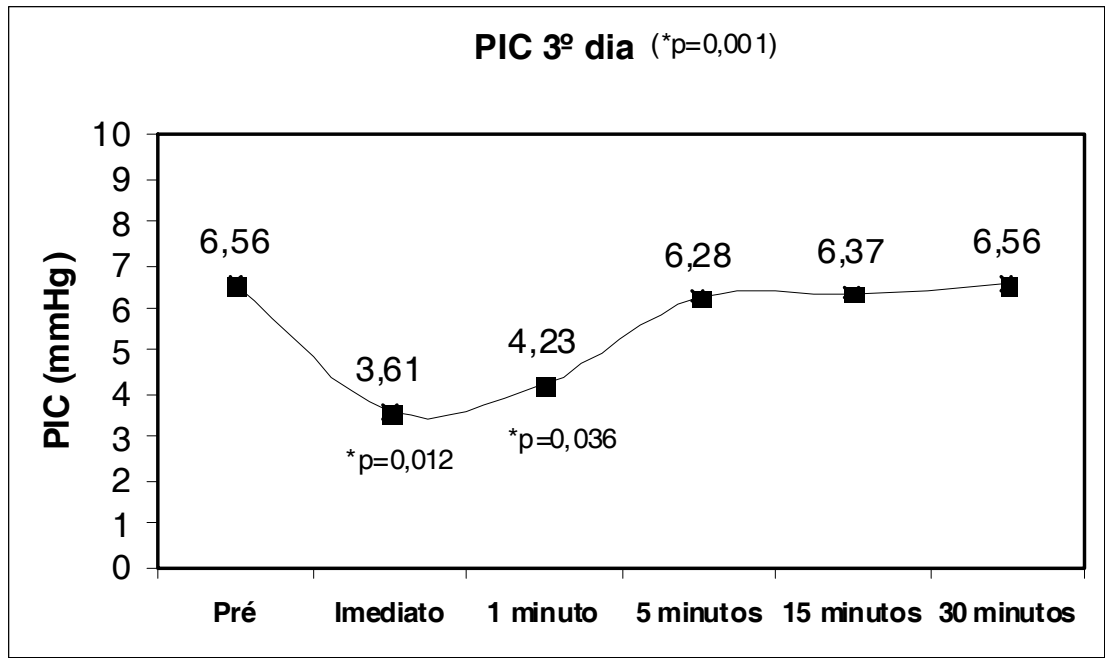

Gráfico 3: Médias da PIC dos 15 pacientes nos distintos momentos da avaliação referente ao 3o dia. 


\section{Discussão}

Este estudo procurou observar a variação da PIC durante manobra de expansão pulmonar de compressão-descompressão torácica, no qual foram avaliados 15 pacientes de ambos os sexos e com média de idade de $29( \pm 13)$ anos. Destes $13 \%$ eram do sexo feminino e $87 \%$, do sexo masculino. Esta afirmação corrobora com os demais estudos, nos quais são descritos que a incidência do TCE predomina em adultos jovens do sexo masculino. ${ }^{36,37}$

Em relação ao trauma, as quedas de nível $(46,7 \%)$ apresentaram maior incidência. Não obstante, outros traumas também ocorrem em índice elevado, tais como colisões $(33,3 \%)$, atropelamento, agressão e corte contuso $(20,1 \%)$. Estes dados estão de acordo com a estatística apresentada pelo Ministério da Saú$\mathrm{de},{ }^{38}$ o qual afirma que a principal causa do TCE no Brasil são as quedas, seguidas pelos acidentes automobilísticos.

A casuística encontrada em estudo realizado por Pitella e Gusmão, ${ }^{29}$ com análise morfológica, macro e microscópica das lesões encefálicas de 120 vítimas fatais de acidente de trânsito, com TCE grave e que evoluíram para HIC, apontou que a grande maioria dos pacientes apresentava como tipo de lesão o hematoma intracerebral. Este vem em consonância com o resultado de nosso estudo, que demonstrou que $66,7 \%$ dos casos apresentaram hematoma intracerebral. Observa-se que a maior incidência das lesões são as que causam hemorragia, independente do tipo, nos casos de TCE grave. Quanto ao tipo de lesão, a incidência maior foi de HED $(40,0 \%)$, na sequência vieram a HSA $(26,7 \%)$, HSD $(26,7 \%)$ e o edema cerebral difuso $(6,7 \%)$.

Nesta pesquisa, adotou-se a manobra de expansão/re-expansão pulmonar, que é um procedimento que aumenta a pressão e/ou volume alveolar, promovendo expansão de unidades alveolares, melhorando o aporte sanguíneo de $\mathrm{O}_{2}$ e posteriormente decréscimo da PIC24, fato que pode justificar a queda da PIC no $1^{\circ}$ minuto pós-manobra, persistindo até o $5^{\circ}$ minuto.

Em estudo realizado por Stiller, ${ }^{39}$ que avaliou diferentes técnicas fisioterapêuticas, a média do aumento da PIC foi menor que $10 \mathrm{mmHg}$. Em nossa pesquisa, durante a manobra fisioterapêutica de expansão pulmonar de compressão-descompressão torácica os resultados da variável PIC demonstraram diferenças entre os períodos pré e pós-manobra. Comparando-se as variações do momento anterior e du- rante a manobra, a PIC apresentou decréscimo em média de 2 a $3 \mathrm{mmHg}$, obtendo respectivamente no $1^{\circ}, 2^{\circ}$ e $3^{\circ}$ dia, $p=0,001, p=0,016$ e $p=0,012$. No momento pré, se comparado com 5 minutos pós-manobra, a média de acréscimo foi de 2 a $3 \mathrm{mmHg}$ com $\mathrm{p}=0,001, \mathrm{p}=0,008$ e $\mathrm{p}=0,036$, respectivamente ao $1^{\circ}, 2^{\circ}$ e $3^{\circ}$ dia. A partir dos 5 minutos pós-manobra, observou-se no início a elevação da PIC gradativamente até 30 minutos, neste momento a pressão atingiu valores próximos dos iniciais em repouso.

Durante a manobra de expansão pulmonar é gerada elevação da pressão transtorácica (PIT), que repercute diretamente nas câmaras cardíacas. Desta forma, a PIC é uma variável dependente da PIT, pressão intra-abdomial (PIA), pressão venosa central e pressão pleural ${ }^{40,41}$ Em contrapartida, as ondas da PIC são mantidas em níveis adequados, devido ao mecanismo de autorregulação.

O Consenso Brasileiro de VM preconiza o uso das manobras respiratórias de manipulação pela fisioterapia, justificando que a grande maioria das técnicas aumenta a PIT e PIC consequentemente ${ }^{42}$ Neste estudo realizado, verificou-se que durante as manobras fisioterapêuticas, a PIT influenciou a pressão alveolar, aumentando a PIC de forma indireta. ${ }^{4}$ Fisiologicamente, durante a inspiração, a PIT torna-se negativa e na expiração, positiva. Porém, tal atividade não é prejudicial, pois mantém os níveis da PIC em equilíbrio, ${ }^{4}$ informação que vem de encontro com os resultados encontrados neste estudo.

Apesar das variações observadas na PIC, seus valores mantiveram-se dentro dos ideais, isto é, abaixo das pressões desejadas para este grupo de pacientes. Quando a PIC é mantida abaixo de $20 \mathrm{mmHg}$, o fluxo sanguíneo cerebral (FSC) e a PPC são otimizados, prevenindo as lesões secundárias e auxiliando na definição da rota de tratamento. ${ }^{43}$ Assim, pode-se considerar que a pressão exercida pela manobra de expansão pulmonar não é suficiente para elevar a PIC acima de $20 \mathrm{mmHg}$, considerando que todos os pacientes estavam estabilizados hemodinamicamente (Gráficos 1, 2, 3). Em contrapartida, considera-se que, apesar da influencia da manobra na PIT, os mecanismos de autorregulação, mecanismos estes fisiológicos, são suficientes para manter a PIC em seus valores de normalidade. No caso da manobra de expansão pulmonar, as alterações hemodinâmicas foram mínimas, viabilizando a aplicação desta manobra em pacientes com TCE grave e com monitorização de PIC. 
Walker ${ }^{44}$ em 1969, iniciou as pesquisas avaliando a atuação da fisioterapia em pacientes com lesões cranianas graves, verificando as alterações hemodinâmicas de compensação e descompensação, durante a drenagem postural, vibração, percussão, RPPI e aspiração endotraqueal. Ele constatou que a fisioterapia pode ser realizada em paciente com quadro de TCE grave e hemodinamicamente estáveis.

De acordo com Koizumi, Pimenta e Sousa ${ }^{45}$ as condutas contra-indicadas em paciente com PIC elevada são a aspiração endotraqueal com duração acima de 10 a 15 segundos, manobra de Valsalva, movimentos que gerem contração isométrica, rotação de cabeça e pescoço. O posicionamento com cabeceira em 30 graus, com cabeça em linha reta em pacientes com TCE grave nas primeiras 24 horas pós trauma reduz a PIC e melhora a PPC. ${ }^{46}$ As condutas contraindicadas acima não foram incluídas no presente estudo, já o posicionamento do paciente no leito durante a aplicação do protocolo respeitou cabeceira com elevação de 30 graus e cabeça em linha reta, observando que a PIC também se manteve abaixo de $20 \mathrm{mmHg}$, conforme descrito no método.

Cerqueira Neto et al. ${ }^{47}$ avaliaram 20 pacientes com TCE grave, os quais se encontravam sedados, curarizados, entubados, em VM em UTI, durante a aplicação de três manobras fisioterapêuticas: vibrocompressão, aumento de fluxo expiratório e aspiração endotraqueal. Foram avaliados a PIC, PPC, PAM, pressão jugular de $\mathrm{O}_{2}$ e saturação jugular de $\mathrm{O}_{2}$. Em seu estudo concluíram que durante as manobras de vibrocompressão e de aumento de fluxo expiratório foram mantidos os parâmetros, enquanto que durante a manobra de aspiração endotraqueal ocorreu elevação da PIC e PAM, retornando aos valores basais após dez minutos. No estudo realizado por Toledo et al. ${ }^{48}$, que avaliou durante três dias consecutivos as variações de PIC durante e após a manobra de vibrocompressão, o resultado não determinou o aumento da PIC em nenhum dos dias avaliados, porém foi observada queda significativa da PIC após a manobra no terceiro dia. Entretanto, durante nosso estudo a PIC apresentou decréscimo no momento da execução da manobra nos três dias, em comparação à medida basal, retornando aos valores próximos dos iniciais progressivamente a partir do $5^{\circ}$ minuto até o $30^{\circ}$ minuto.

Em estudo realizado por Thiesen et al. ${ }^{4}$, avaliando 35 pacientes, dispostos em 3 grupos (PIC de 0 $10 \mathrm{mmHg}$; PIC de 11-20 mmHg; PIC de 21-30 $\mathrm{mmHg}$ ), foram eleitas 8 condutas fisioterapêuticas: pressão manual com vibração manual costal; pressão expiratória com vibração manual diafragmática; drenagem postural com tapotagem; pressão manual expiratória com descompressão diafragmática; pressão manual expiratória com descompressão manual costal; respiração costal contrariada; respiração localizada; e aspiração endotraqueal. As medidas da PIC, no estudo acima, foram realizadas no $1^{\circ}$ e $30^{\circ}$ minuto, após a realização de todas as manobras. $\mathrm{O}$ resultado demonstra que, depois 30 minutos do procedimento de aspiração endotraqueal, a PIC apresentou decréscimo de $1 \mathrm{a} 2 \mathrm{mmHg}$, possivelmente pela melhora da ventilação pulmonar. No entanto, as demais manobras não apresentaram variações significativas. Os valores encontrados no estudo de Thiesen et al. aproximam-se dos resultados deste estudo. A indicação desses resultados é o manuseio do paciente com TCE grave e com monitorização de PIC, no caso da manobra de expansão pulmonar. Destaca-se que seu principal objetivo é facilitar a relação ventilação perfusão (V/Q) e elevar as pressões parciais de oxigênio $\left(\mathrm{O}_{2}\right)$, consequentemente, ocasionando uma vasoconstrição, que diminui o FSC e a PIC. ${ }^{16}$ Este fato pode justificar o decréscimo da PIC, no momento da realização da manobra, o qual persiste por cerca de 1 minuto, devido ao melhor aporte de $\mathrm{O}_{2}$ intracerebral, sendo este otimizado devido ao recrutamento alveolar.

Thiesen et al. afirmam que as manobras fisioterapêuticas respiratórias não alteram a PIC. Desta forma, podem ser realizadas com segurança em pacientes com TCE grave e com os valores de PIC em até $30 \mathrm{mmHg}$. Esta conduta diferencia-se daquela aplicada em nosso estudo, porque o local onde foi realizada a pesquisa adota como protocolo da PIC ideal entre 0-20 mmHg. Acima desta pressão, no protocolo do Hospital Universitário Cajuru, considera-se HIC e por isso o atendimento deste paciente não é realizado, devido ao risco de agravamento do quadro clínico.

O papel do fisioterapeuta na UTI é indispensável no manejo de pacientes graves, principalmente em casos que contenham risco de evolução eminente de HIC. De acordo com Mehanna, Tanaka e Duarte ${ }^{9}$ e Paraíbuna e Marx $^{49}$ deve-se observar a variação abrupta da PIC para não agravar o quadro clínico do paciente por meio das manobras fisioterapêuticas. Estes resultados parecem indicar a necessidade de aplicação das manobras fisioterapêuticas em pacientes com risco de HIC.

Além da verificação constante da PIC, outros dados devem ser analisados para uma melhor inter- 
venção como os valores de PAM e da PPC, dados que são geralmente correlacionados com a PIC.

As variações da PAM foram mínimas durante e após a manobra de compressão-descompressão torácica neste grupo estudado. As mesmas mantiveramse dentro dos valores de normalidades de acordo com a patologia. Os valores desejados da PAM nos casos com risco de HIC são de 60 a $180 \mathrm{mmHg} .{ }^{4-9}$ A média obtida foi de $81,42( \pm 11,63) \mathrm{mmHg}$. Nos casos de hipotensão arterial e hipertensão arterial, a variação da PAM é prejudicial ao cérebro, portanto, deve ser evitada. ${ }^{3,19} \mathrm{O}$ uso de drogas vasoativas muitas vezes é indicado para manter a PAM nos valores ideais. Contanto, outro fato importante é que o decréscimo da PAM é induzido pela administração de sedativos adicionais. ${ }^{19}$ Durante nosso estudo, os pacientes estavam com sedação contínua, sendo em alguns deles necessário a utilização de drogas vasoativas para manter adequada a PAM.

$\mathrm{Na}$ literatura, os valores recomendados para a PPC devem ser acima de $60 \mathrm{mmHg}^{10}$. Em nosso estudo a PPC se manteve entre 66,22 $( \pm 13,03)$ $\mathrm{mmHg}$ e $76,13( \pm 15,11) \mathrm{mmHg}$. Este resultado é corroborado com um estudo de Jull et al. ${ }^{16}$ onde foram avaliados 427 pacientes pertencentes ao grupo de risco admitido em UTI. Existe risco de piora do quadro clínico quando a PPC se encontra abaixo de $60 \mathrm{mmHg}$. Neste caso, a PPC poderia ser reduzida tanto pelo decréscimo da PAM ou como pela elevação da PIC. ${ }^{3}$

Os valores da PPC e da PAM são semelhantes, devido à direta correlação entre estes dados (PPC: PAM-PIC), em que a PPC é diretamente proporcional à PAM. $\mathrm{O}$ aumento da PIC pode ser decorrente do aumento do volume do sangue cerebral, devido à queda da PPC abaixo do seu limite. Os mecanismos autorreguladores do FSC, que operam dentro de 1 minuto, induzem a vasodilatação que, por sua vez, aumenta a PIC. ${ }^{20}$ No presente estudo, a PIC apresentou decréscimo no momento imediato da aplicação da manobra, retornando aos valores próximos aos iniciais após 5 minutos. Quando a PIC e a PAM são man- tidas dentro dos valores de normalidades, espera-se que a PPC seja ideal com aporte sanguíneo cerebral desejado, sendo que neste estudo a média de PPC obtida foi de $72,80( \pm 18,30) \mathrm{mmHg}$.

\section{Conclusão}

Percebeu-se, com esta pesquisa, que a manobra de compressão-descompressão torácica, isoladamente, não promoveu neste estudo alteração hemodinâmica cerebral nas variáveis PIC e nas variáveis associadas da PAM e PPC. Também se constatou redução significativa da PIC no momento imediato da realização da manobra, com decréscimo entre 2 e 3 $\mathrm{mmHg}$, persistindo no $1^{\circ}$ minuto depois da manobra e, posteriormente, retornando no $5^{\circ}$ minuto a valores próximos aos iniciais. Desta forma, a manobra de compressão-descompressão torácica não gerou elevação da PIC para desenvolver complicações deletérias em pacientes hemodinamicamente estáveis analisados neste estudo. Contudo, já os valores da PPC e PAM, mantiveram-se estáveis durante todo o protocolo com valores ideais ou próximos dos ideais.

No grupo avaliado, a técnica pulmonar utilizada trouxe efeitos positivos para os pacientes com TCE grave, sedados, em VM e com monitorização de PIC. Portanto, a manobra de compressão-descompressão apresentou-se como uma conduta adequada em pacientes com TCE em unidade crítica hospitalar, ajudando assim, na reabilitação fisioterapêutica pulmonar.

\section{Agradecimentos}

Ao Hospital Universitário Cajuru, pela disponibilidade do campo de pesquisa, a Doutora Mabel Kawasaki, a Fisioterapeuta Lilianne Pertschie, a todos os plantonistas da UTI, equipe da neurocirurgia, equipe de enfermagem e a todos que colaboraram para a realização desta pesquisa, sobretudo aos pacientes e familiares, pois sem eles não seria possível à conclusão deste estudo. 


\begin{abstract}
In physical therapy, there are specific means available for rehabilitation in the ICU, as well as for patients with severe traumatic brain injury (TBI). Among the available techniques, the pulmonary expansion maneuver, a kinesiotherapy technique aimed at mobilizing lung secretions, preventing and treating atelectasis. Model of the study: The study was classified as field research (experimental), based on assessment of the ICP during and after pulmonary expansion maneuver. Objective: To verify how the pulmonary expansion therapy affects intracranial pressure (ICP) in patients with severe TBI. Methodology: The study was approved by the Ethics and Research Committee of the PUCPR (opinion 1455). It was carried out at the unity of intensive therapy (ICU) in the Cajuru University Hospital, Curitiba-PR. Fifteen severe TBI patients of both genders participated in the study, their ages ranging between 18 and 50 years. The monitored variables were ICP, mean arterial pressure (MAP) and brain perfusion pressure (BPP). Results: During the application of the research protocol it was observed that MAP and the BPP remained within or close to normal range with slight changes, while ICP increased approximately $1 \mathrm{mmHg}$ then returning to baseline values. Mean ICP in the 1 st, 2 nd and 3 rd days were $5,42( \pm 4,69) \mathrm{mmHg}, 6,71$ $( \pm 6,84) \mathrm{mmHg}$ and $5,60( \pm 4,33) \mathrm{mmHg}$ with statistical significance of $p<0,001, p=0,008$ and $p=0,001$ respectively. Conclusions: The pulmonary expansion maneuver may be performed in severe TBI patients who are hemodynamically stable and have a baseline ICP below $20 \mathrm{mmHg}$.
\end{abstract}

Keywords: Physical Therapy (Specialty). Respiratory Therapy. Intracranial Pressure. Traumatic Brain Injury. Physical Therapy Department, Hospital.

\section{Referências Bibliográficas}

1. Koizumi MS. Monitorização da pressão intracraniana. Rev Esc Enferm USP. 1981; 15: 147-54.

2. Lundberg N. Continuous recording and control of ventricular fluid pressure in neurosurgical practice. Acta psychiat neurol scand. 1960; 36 (Suppl 149).

3. Hlatky R, Valadka AB, Robertson CS. Intracranial hypertension and cerebral ischemia after severe traumatic brain injury. Neurosurg focus. 2003; 14: 1-4.

4. Thiesen RA, Dragosavac D, Roquejani AC, Falcão ALE, Araujo S, Dantas Filho VP, et al. Influência da fisioterapia respiratória na pressão intracraniana em pacientes com traumatismo cranioencefálico grave. Arq neuropsiquiatr. 2005; 63: 110-3.

5. Perreira RSS, Mello PA, Horta MP. Monitorização contínua da pressão intracraniana no manuseio do traumatismo crânioencefálico grave. J bras med. 1984; 47: 57-63.

6. Valentin A, Lang T, Karnik R, Ammerer HP, Ploder J, Slany J. Intracranial pressure monitoring and case mix-adjusted mortality in, intracranial hemorrhage. Crit care med. 2003; 31: 1539-42.

7. Brandt RA, Féres Júnior $H$, Ribas GL, Capone Neto A. Traumatismo cranioencéfalico. In: Knobel E. Condutas no paciente grave. $2^{\mathrm{a}}$ ed. São Paulo: Atheneu; 2006. p. 1261-82.

8. Cecil RL, Smith LH, Bennett JC. Tratado de medicina intensivo. $19^{a}$ ed. Rio de Janeiro: Guanabara Koogan; 1993. p. 2353-9.

9. Mehanna A, Tanaka CM, Halina D. Relato de caso dos possíveis implicações das manobras fisioterapêuticas sobre a pressão intracraniana paciente com aneurisma intracraniano. Fisioter mov. 2000; 12: 89-97.

10. Marin JJ, Heler APW. Terapia intensiva. $2^{\underline{a}}$ ed. São Paulo: Manole; 1999. p. 545-58.

11. Joseph AK, Dutton RP, Aarobi B, Scalea TM. Decompressive laparotomy to treat intractable intracranial hypertension after traumatic brain injury. J trauma. 2004; 57: 687-95.
12. Andrade AF, Brock RS, Shu EBS, Figueiredo EG, Marino Júnior R. Terapia intensiva no trauma de crânio. In: Freire E. Trauma doença do século. São Paulo: Guanabara Koogan; 2000. p.1151-63.

13. Capone Neto A, Ferraz AC, Brandt RA, Ribas GC. Hipertensão no traumatismo cranioencefálico. In: Knobel E. Condutas no Paciente Grave. 3aㅡ ed. São Paulo: Atheneu; 2006. p. 847-56.

14. Capone Neto A, Joaquim MAS, Lucas FJC, Machado FS. Monitorização neurológica intensiva. In: Knobel E. Condutas no Paciente Grave. $3^{\mathrm{a}}$ ed. São Paulo: Atheneu; 2006. p. 85768.

15. Jennett WB, Harper AM, Miller JD, Rowan JO. Relation between cerebral blood-flow and cerebral perfusion pressure. $\mathrm{Br}$ j surg. 1970; 57(5): 390.

16. Jull N, Morri GF, Marshall SB, Marshall LF. Intracranial hypertension and cerebral perfusion pressure: influence on neurological deterioration and outcome in severe head injury. J neurosurg. 2000; 92:1-6.

17. Portella G, Cormio M, Citerio G. Continuos cerebral compliance monitoring in severe head injury: its relationship with intracranial pressure and cerebral perfusion pressure. Acta neurochir. 2002; (Suppl 81):173-5.

18. Mack WJ, King RG, Ducruet AF, Kreiter K, Mocco J, Maghoub $A$, et al. Intracranial pressure following aneurysmal subarachnoid hemorrhge: monitoring practices and outcome data. Neurosurg focus. 2003;14: 1-5.

19. Ersson U, Carlson H, Mellstrom A, Pontém U, Hedstrand U, Jakobsson S. Observations on intracranial dynamics during respiraty physiotherapy in unconscious neurosurgical patients. Acta anaesthesiol scand. 1990; 34: 99-103.

20. Barbosa AP, Cabral SA. Novas terapias para hipertensão endocranianas. J pediatr. (Rio J.). 2003;79 (Suppl 2): 139-48.

21. Brimioulle S, Moraine JJ, Norrenberg D, Kahn RJ. Effects of positionig and exercise on intracranial pressure a neurosurgincal intensive care unit. Phys ther. 1997; 77: 1682-9.

22. Kisner C, Colly LA. Exercícios Terapêuticos Fundamentos e Técnicas. $3^{\underline{a}}$ ed. São Paulo: Manole; 1998. p. 654-5. 
23. Ntoumenopoulos G, Presneill JJ, McElholum M, Cade JF. Chestphysiotherapy for the prevention of ventilator-associated pneumonia. Intensive care med. 2002; 28: 850-6.

24. Jerre G, Beraldo MA, Silva TJ, Gastaldi A, Kondo C, Leme F, et al. Fisioterapia no paciente sob ventilação mecânica. III Consenso Brasileiro de Ventilação Mecânica. Rev bras ter intensiva. 2007; 19: 399-407.

25. Malbouisson LMS, Humberto F, Rodrigues RR, Carmona MJ, Auler Júnior JOC. Atelectasias durante anestesia: Fisiopatologia e tratamento. Rev bras anestesiol. 2008; 58: 73-83.

26. Johnston C, Carvalho WB. Atelectasias em pediatria: mecanismos, diagnósticos e tratamento. Rev Assoc Med Bras. 2008; 54: 455-460.

27. Azeredo CAC, Nemer SN, Azeredo LM. Fisioterapia respiratória em UTI. 1 $1^{a}$ ed. São Paulo: Manole, 1998. p.197-201.

28. Unoki T, Kawasaki Y, Mizutani T, Fujino Y, Yanagisawa Y, Ishimatsu S. Effects of expiratory rib-cage compression on oxygenation, ventilation, and airway-secretion removal in patients receiving mechanical ventilation. Respir care. 2005; 50: $1430-7$.

29. Pittella JEH, Gusmão SS. Hipertensão intracraniana em vítimas fatais de acidente de trânsito. Arq neuropsiquiatr. 1999; 57: 843-7.

30. McCarren B, Alisson JA, Herbert RD. Manual vibration increases expiratory flow rate via increased intrapleural pressure in healthy adults: an experimental study. Aust j physiother. 2006; 52: 267-71.

31. McCarren B, Alisson JA, Herbert RD. Vibration an its effect on the respiratory system. Aust j physiother. 2006, 52: 39-43.

32. McCarren B, Alisson JA, Herbert RD. The use of vibration in public hospitals in Australia. Physiother theory pract. 2003; 19: 87-98.

33. Hess DR. The evidence for secretion Clearance Techniques. Respir care. 2001; 46: 1276-92.

34. Presto B, Presto LDN. Fisioterapia respiratória uma nova visão. Rio de Janeiro: Bruno Presto; 2003. p. 69-71.

35. Azeredo CAC. Fisioterapia respiratória no hospital geral. São Paulo: Manole; 2000. p. 451-70.

36. Souza JM, Reis M, Souto AA. D. Epidemiologia do traumatismo de crânio. In: Freire E. Trauma: a doença do século. $2 \mathrm{v}$. São Paulo: Guanabara Koogan; 2001. p. 1197-211.

37. Melo JRT, Silva RA, Moreira ED. Características dos pacientes com trauma cranioencefálico na cidade do Salvador, Bahia, Brasil. Arq neuropsiquiatr. 2004; 62: 711-5.
38. Ministério da Saúde. Informações de Saúde - Mortalidade (2004) [última atualização] 05/2004 [citado em 08/2007]. Disponível em: http://www.datasus.gov.br/.

39. Stiller K. Physiotherapy in Intensive Care: towards an evidence-based practive. Chest. 2000; 118: 1804.

40. Bloomfield GL, Ridings PC, Blocher CR, Marmarou A, Sugerman HJ. A proposed relationship between increased intra-abdominal, intrathoracic, and intracranial pressure. Crit care med. 1997; 25: 496-502.

41. Villaça MP, Mantovani M. Comportamento da pressão intracraniana, da perfusão cerebral e dos parâmetros hemodinâmica durante a síndrome do compartimento abdominal em cães. Rev Col Bra Cir. 2006; 33: 211-4.

42. Dias MD. Ventilação mecânica no trauma. J pneumol. 2000; 26 (Suppl 2): 42-53.

43. Giugno KM, Maia TR, Kunrath CL, Bizzi JJ. Tratamento de hipertensão intracraniana. J pediatr. (Rio J.). 2003;79(4): 28796.

44. Walker J. Physiotherapy for patients with cranial lesions. Physiotherapy. 1969; 55: 98-9.

45. Koizumi MS, Pimenta CAM, Sousa RMC. Condutas de enfermagem e aumento da pressão intracraniana. Rev gaúch enferm.1989; 15: 147-54.

46. Ng I, Lim J, Wong HB. Effects of head posture on cerebral hemodynamics: Its influences on intracranial pressure, cerebral perfusion pressure, and cerebral oxygenation. Neurosurgery. 2004; 54: 593-8.

47. Cerqueira Neto ML, Moura A, Aquim EE, Oliveira MC, Cerqueira TCF, Silva RA, Rea Neto A. Efeitos das manobras fisioterapêuticas respiratórias de vibrocompressão, aumento de fluxo expiratório e aspiração endotraqueal sobre a hemodinâmica cerebral em pacientes com traumatismo cranioencefálico. Rev bras fisioter. 2006; (Suplemento): 24

48. Toledo C, Garrido C, Troncoso E, Lobo SM. Efeitos da fisioterapia respiratória na pressão intracraniana e na pressão de perfusão cerebral no traumatismo cranioencefálico grave. Rev bras ter intensiva. 2008, 20(4): 339-343.

49. Paraíbuna CC, Marx CB. Fisioterapia intensiva e otimização ventilatória no paciente com hipertensão intracraniana aguda. Rev Intensiva, 2005; 3: 93-9. 